\title{
Sturmian Sequences and Invertible Substitutions
}

\author{
Li Peng" and Bo Tan $\mathbb{\beta}$ \\ Department of Mathematics, Huazhong University of Science and Technology, Wuhan 430074, P.R.CHINA
}

received $27^{\text {th }}$ December 2009, revised 25 th June 2011, accepted $27^{\text {th }}$ June 2011.

It is known that a Sturmian sequence $S$ can be defined as a coding of the orbit of $\rho$ (called the intercept of $S$ ) under a rotation of irrational angle $\alpha$ (called the slope). On the other hand, a fixed point of an invertible substitution is Sturmian. Naturally, there are two interrelated questions: (1) Given an invertible substitution, we know that its fixed point is Sturmian. What is the slope and intercept? (2) Which kind of Sturmian sequences can be fixed by certain non-trivial invertible substitutions? In this paper we give a unified treatment to the two questions. We remark that though the results are known, our proof is very elementary and concise.

Keywords: Sturmian sequence, Invertible substitution

\section{Introduction}

Sturmian sequences are infinite words over a binary alphabet with minimal complexity, and these sequences admit several equivalent definitions under different names. In this paper, we adopt the following definition.

Let $\alpha \in[0,1] \backslash \mathbb{Q}$ and $\rho \in \mathbb{R}$. We define, for $n \in \mathbb{N}=\{0,1,2, \cdots\}$,

$$
\begin{aligned}
& S_{\alpha, \rho}(n)=\lfloor\alpha(n+1)+\rho\rfloor-\lfloor\alpha n+\rho\rfloor, \\
& S_{\alpha, \rho}^{\prime}(n)=\lceil\alpha(n+1)+\rho\rceil-\lceil\alpha n+\rho\rceil,
\end{aligned}
$$

and call $S$ (resp. $S^{\prime}$ ) the lower (resp. upper) Sturmian sequence with slope $\alpha$ and intercept $\rho$. Obviously, we can assume that $\rho \in \mathbb{S}:=\mathbb{R} / \mathbb{Z}$.

It is easy to see that Sturmian sequences are over the alphabet $A=\{0,1\}$. In other words, $S, S^{\prime} \in A^{\mathbb{N}}$, where $A^{\mathbb{N}}$ is the set of infinite sequences over $A$.

Sturmian sequences are extensively studied by many authors, and excellent descriptions can be found in Chapter 2 of [5] by J. Berstel \& P. Séébold, and in Chapter 6 of [6] by P. Arnoux.

Let $A=\{0,1\}$ be the alphabet, and $A^{*}$ (resp. $F(A)$ ) stand for the free monoid (resp. free group) generated by $A$. The empty word $\varepsilon$ is their identity element. For $u \in A^{*},|u|$ denotes the length of $u$ (thus

\footnotetext{
${ }^{\dagger}$ Supported by NSFC 10631040 and 10901066.

‡Email: pengandtan@yahoo.com

$\S$ Corresponding author. Email: bo_tan@163.com

1365-8050 @ 2011 Discrete Mathematics and Theoretical Computer Science (DMTCS), Nancy, France
} 
$|\varepsilon|=0$ ), and $|u|_{i}$ denotes the number of occurrences of the letter $i$ in the word $u$. The mirror of a word $w=w_{0} w_{1} \cdots w_{n-1}$, where $w_{i} \in A$, is the word $w_{n-1} \cdots w_{1} w_{0}$.

A morphism $\tau: A^{*} \rightarrow A^{*}$ is called a substitution of $A^{*}$. Two substitutions $\tau$ and $\sigma$ are said to be conjugate, if there is a word $w \in A^{*}$ such that $\tau(i) w=w \sigma(i)$ for any $i \in A$; or vice versa.

If a substitution $\tau$ can be extended to an automorphism of $F(A)$, we say that it is invertible. In particular, an invertible substitution $\tau$ is non-erasing, that is, both $\tau(0)$ and $\tau(1)$ are different from $\varepsilon$.

On the structure of invertible substitutions, Wen \& Wen [9] showed that any invertible substitution is a composition of the following three substitutions:

$$
\pi=(1,0), \quad \sigma=(01,1) \quad \text { and } \quad \phi=(10,0),
$$

where the notation $(u, v)$ denotes the substitution $0 \mapsto u, 1 \mapsto v$.

A substitution $\tau$ can also be extended to a mapping of $A^{\mathbb{N}}$. And $\tau$ is called Sturmian if $\tau(\xi)$ is a Sturmian sequence for any Sturmian sequence $\xi$. Mignosi \& Séébold [7] proved that a Sturmian substitution is also a composition of the above three substitutions. Therefore, a substitution is Sturmian if and only if it is invertible.

A substitution is called non-trivial if it is not the identity. From the above results, we know that, given a non-trivial invertible substitution $\tau$, if $\xi$ is a fixed point of $\tau$ (i.e. $\tau(\xi)=\xi$ ), then $\xi$ is a Sturmian sequence (with the following exceptions of non-primitive substitutions: $\left(01^{n}, 1\right)$ which fixes $01^{\infty}$, and $\left(0,10^{n}\right)$ which fixes $10^{\infty}$ ).

Naturally, we ask the following two interrelated questions:

QUESTION 1. Given an invertible substitution with a fixed point, what is the slope and the intercept?

QUESTION 2. Which kind of Sturmian sequence can be fixed by certain non-trivial substitutions?

The first question was tackled in Tan \& Wen [8]. In fact, the slope is just the ratio of the frequencies of the two letters in the fixed point, and it is easy to determine via the substitution matrix. And the intercept is obtained by a very delicate comparison of the intercepts between the substitutions in a same conjugate class.

Yasutomi [10] gave a complete answer to the second question, by considering how the three elementary invertible substitutions change the slope and intercept of a Sturmian sequence. Later, Baláži, Masáková \& Pelantová [1] and Berthé, Ei, Ito \& Rao [2] gave alternative proofs of the characterization via the cutand-project scheme and the Rauzy fractal respectively. These proofs are somewhat technical and lengthy.

In this paper, we recall a characterization of the invertible substitution, and then provide a unified treatment to the two questions. With help of the characterization, our proofs are very elementary and concise.

\section{Auxiliary Results}

The shift function is the mapping $T: A^{\mathbb{N}} \rightarrow A^{\mathbb{N}}$ defined by $T\left(x_{0} x_{1} x_{2} x_{3} \cdots\right)=x_{1} x_{2} x_{3} \cdots$. And the shift changes the intercept of a Sturmian sequence in an obvious way.

Lemma 2.1 We have that $T S_{\alpha, \rho}=S_{\alpha, \alpha+\rho}, \quad T S_{\alpha, \rho}^{\prime}=S_{\alpha, \alpha+\rho}^{\prime}$.

We mention several special cases: $S_{\alpha, \alpha}=S_{\alpha, \alpha}^{\prime}$, called the characteristic sequence and also denoted by $C_{\alpha} ; S_{\alpha, 0}=0 C_{\alpha}, S_{\alpha, 0}^{\prime}=1 C_{\alpha} ; S_{\alpha, 1-\alpha}=10 C_{\alpha}, S_{\alpha, 1-\alpha}^{\prime}=01 C_{\alpha}$.

The next lemma is also readily checked. 
Lemma 2.2 If $\rho_{n} \rightarrow \rho+($ in $\mathbb{S})$, that is, $\rho_{n}$ tends to $\rho$ in $\mathbb{S}$ anticlockwisely, then $S_{\alpha, \rho_{n}} \rightarrow S_{\alpha, \rho}^{\prime}$ and $S_{\alpha, \rho_{n}}^{\prime} \rightarrow S_{\alpha, \rho}^{\prime} ;$ If $\rho_{n} \rightarrow \rho-($ in $\mathbb{S})$, then $S_{\alpha, \rho_{n}} \rightarrow S_{\alpha, \rho}$ and $S_{\alpha, \rho_{n}}^{\prime} \rightarrow S_{\alpha, \rho}$.

The following characterization of invertible substitutions is shown in [8], which is essentially equivalent to the geometrical representation [4]. The reader is referred to these papers for more details.

Theorem 2.3 Let $\tau$ be a substitution. Then $\tau$ is invertible if and only if there exist $u$ and $v \in A^{*}$, such that

$$
\text { either }\left\{\begin{array} { l } 
{ \tau ( 0 1 ) = u 0 1 v } \\
{ \tau ( 1 0 ) = u 1 0 v }
\end{array} \text { or } \left\{\begin{array}{l}
\tau(01)=u 10 v \\
\tau(10)=u 01 v
\end{array} .\right.\right.
$$

\section{The slope and intercept of a substitutive Sturmian sequence}

Let $\tau$ be an invertible substitution with a fixed point $\xi$. The substitution matrix of $\tau$ is defined as $M=$ $\left(|\tau(j)|_{i}\right)_{i, j=0,1}$. We will assume that $M$ is primitive, and its Perron-Frobenius eigenvalue is denoted by $\lambda$. The slope and intercept of $\xi$ are denoted by $\alpha$ and $\rho$ respectively.

In this section, given an invertible substitution $\tau$, we calculate the slope $\alpha$ and intercept $\rho$. It is easy to determine $\alpha$ via the substitution matrix $M$.

Lemma 3.1 We have that

$$
M\left(\begin{array}{c}
1-\alpha \\
\alpha
\end{array}\right)=\lambda\left(\begin{array}{c}
1-\alpha \\
\alpha
\end{array}\right)
$$

The following theorem on the intercept is due to [8] (under a big guise).

Theorem 3.2 Let $\tau$ be a primitive invertible substitution with a fixed point. With the above notations, we have that

$$
\rho=1-\alpha-\frac{1}{1-\bar{\lambda}}(\alpha, \alpha-1) P(u)=1-\alpha-\frac{1}{1-\bar{\lambda}}(|u| \alpha-\lfloor(|u|+1) \alpha\rfloor),
$$

where $P(u)=\left(|u|_{0},|u|_{1}\right)^{T}$ is the Parikh vector of $u ; \bar{\lambda}$ is the Galois conjugate of $\lambda$; and $u$ is the word associated with $\tau$ as in Theorem 2.3

Proof: For any $n \in \mathbb{N}$, let $\left(u_{n}, v_{n}\right)$ be the pair associated with $\tau^{n}$, that is,

Then

$$
\text { either }\left\{\begin{array} { l } 
{ \tau ^ { n } ( 0 1 ) = u _ { n } 0 1 v _ { n } } \\
{ \tau ^ { n } ( 1 0 ) = u _ { n } 1 0 v _ { n } }
\end{array} \quad \text { or } \left\{\begin{array}{l}
\tau^{n}(01)=u_{n} 10 v_{n} \\
\tau^{n}(10)=u_{n} 01 v_{n}
\end{array}\right.\right.
$$

$$
u_{n}=\tau^{n-1}(u) \tau^{n-2}(u) \cdots \tau(u) u ; \quad v_{n}=v \tau(v) \cdots \tau^{n-2}(v) \tau^{n-1}(v)
$$

Thus, if $v \neq \epsilon$, the $\operatorname{limit} \lim _{n \rightarrow \infty} v_{n}$ exists, say $S$. And then both $0 S$ and $1 S$ are Sturmian, which implies that $S=C_{\alpha}$ (the characteristic word).

Let $\xi_{n}:=u_{n} 10 C_{\alpha} \rightarrow \eta$ and $\xi_{n}^{\prime}:=u_{n} 01 C_{\alpha} \rightarrow \eta^{\prime}$ as $n \rightarrow \infty$. Then both $\eta$ and $\eta^{\prime}$ are fixed points of $\tau$. Let us remark that the conclusion also holds when $v=\epsilon$. Moreover, if $u \neq \epsilon$, then $\eta=\eta^{\prime}$; if $u=\epsilon$, then $\eta=10 C_{\alpha}$, and $\eta^{\prime}=01 C_{\alpha}$.

Now by Lemma 2.1. $\xi_{n}=u_{n} 10 C_{\alpha}=S_{\alpha,-\left(\left|u_{n}\right|+1\right) \alpha}$, and $\xi_{n}^{\prime}=u_{n} 01 C_{\alpha}=S_{\alpha,-\left(\left|u_{n}\right|+1\right) \alpha}^{\prime}$. So

$$
\rho=\lim -\left(\left|u_{n}\right|+1\right) \alpha \quad(\text { in } \mathbb{S}) .
$$


From Eq. 3 we infer that $\left|u_{n}\right|=\left|\tau^{n-1}(u) \cdots \tau(u) u\right|=(1,1)\left(M^{n-1}+\cdots+M+I\right) P(u)$. Since the left and right eigenvector associated with distinct eigenvalues respectively are orthogonal to each other, we obtain from Eq. (1) that $(\alpha, \alpha-1) M=\bar{\lambda}(\alpha, \alpha-1)$. So,

$$
\begin{aligned}
-\left(\left|u_{n}\right|+1\right) \alpha & =-\alpha-(\alpha, \alpha)\left(M^{n-1}+\cdots+M+I\right) P(u) \\
& \left.=1-\alpha-(\alpha, \alpha-1)\left(M^{n-1}+\cdots+M+I\right) P(u) \quad \text { (in } \mathbb{S}\right) \\
& =1-\alpha-(\alpha, \alpha-1)\left(\bar{\lambda}^{n-1}+\cdots+\bar{\lambda}+1\right) P(u) \\
& \rightarrow 1-\alpha-\frac{1}{1-\bar{\lambda}}(\alpha, \alpha-1) P(u)=1-\alpha-(\alpha, \alpha-1)(I-M)^{-1} P(u) .
\end{aligned}
$$

Since both $u 0$ and $u 1$ are factors, the mirror word of $u$ is a prefix of $C_{\alpha}=s_{0} s_{1} s_{2} \cdots$, so $|u|_{1}=$ $\sum_{0}^{|u|-1} s_{i}=\lfloor(|u|+1) \alpha\rfloor-\lfloor\alpha\rfloor=\lfloor(|u|+1) \alpha\rfloor$, and $P(u)=(|u|-\lfloor(|u|+1) \alpha\rfloor,\lfloor(|u|+1) \alpha\rfloor)^{T}$. Therefore,

$$
\rho=1-\alpha-\frac{1}{1-\bar{\lambda}}(\alpha, \alpha-1) P(u)=1-\alpha-\frac{1}{1-\bar{\lambda}}(|u| \alpha-\lfloor(|u|+1) \alpha\rfloor) .
$$

It remains to show that $\rho \in[0,1]:$ Since

$$
\rho_{n}:=1-\alpha-(\alpha, \alpha-1) P\left(u_{n}\right) \rightarrow \rho \quad(\text { in } \mathbb{R}),
$$

we only need to show $\rho_{n} \in(0,1)$. This is the case because $(\alpha, \alpha-1) P\left(u_{n}\right)=\left|u_{n}\right| \alpha-\left\lfloor\left(\left|u_{n}\right|+1\right) \alpha\right\rfloor$, and $\rho_{n}=1-\left(\left|u_{n}\right|+1\right) \alpha+\left\lfloor\left(\left|u_{n}\right|+1\right) \alpha\right\rfloor \in(0,1)$.

Let $\tau$ be invertible with $\operatorname{det} M=1$. Denote $\|\tau\|=\|M\|=|\tau(01)|$. If $\varphi$ is a conjugate substitution of $\tau$, then they have the same substitution matrix (thus have the same slope $\alpha$ ), and the same word $v u$. Moreover, when $\varphi$ runs through $\operatorname{Con}(\tau)$, the class of conjugate substitutions of $\tau$, the length of the corresponding word $u$ runs through all the integers between 0 and $\|\tau\|-2$. so, by Theorem 3.2, we have (recalling that $\rho(\varphi)$ is the intercept of the fixed point of $\rho$ )

$$
\{\rho(\varphi) ; \varphi \in \operatorname{Con}(\tau)\}=\left\{1-\alpha-\frac{1}{1-\bar{\lambda}}(n \alpha-\lfloor(n+1) \alpha\rfloor) ; n=0,1, \cdots,\|\tau\|-2\right\} .
$$

Let us consider some special cases:

$$
\begin{aligned}
u=\epsilon . & \text { Then } \rho(\varphi)=1-\alpha, \text { and } \varphi \text { has two fixed points, namely } 01 C_{\alpha} \text { and } 10 C_{\alpha} . \\
v=\epsilon . & \begin{array}{r}
\text { Then } \varphi(01)=u 01, P(u)=(M-I)(1,1)^{T}, \text { and } \rho=1-\alpha-(\alpha, \alpha-1)(I- \\
M)^{-1} P(u)=\alpha . \text { Whence } \varphi \text { fixes } C_{\alpha} .
\end{array} \\
|u|=|\tau(0)|-1 . & \begin{array}{l}
\text { Then, Taking the lengths into account in the formula } \varphi(01)=u 01 v, \text { we get } \\
\varphi(0)=u 0, \text { so } P(u)=(M-I)(1,0)^{T} \text { and } \rho=1 . \text { Whence } \varphi \text { fixes } 1 C_{\alpha} .
\end{array} \\
|u|=|\tau(1)|-1 . & \text { Then } P(u)=(M-I)(0,1)^{T}, \rho=0, \text { and } \varphi \text { fixes } 0 C_{\alpha} .
\end{aligned}
$$

Now $(\alpha, \rho)$ is already obtained for $\tau$. It remains to distinguish that the fixed point is $S_{\alpha, \rho}$ or $S_{\alpha, \rho}^{\prime}$.

- if $\rho \notin \mathbb{N}-\mathbb{N} \alpha$, then $S_{\alpha, \rho}=S_{\alpha, \rho}^{\prime}$; 
- if $\rho \in \mathbb{N}-\mathbb{N} \alpha$, then, recalling $\rho=1-\alpha+(\alpha, \alpha-1)(M-I)^{-1} P(u)$, we have $(M-I)^{-1} P(u) \in \mathbb{Z}^{2}$. So $(M-I)^{-1} P(u) \in\left\{(0,0)^{T},(0,1)^{T},(1,0)^{T},(1,1)^{T}\right\}$, thus

$$
\rho \in\{1-\alpha, 0,1, \alpha\} .
$$

And all these cases are already studied.

As a byproduct, we have shown the following well-known result.

Proposition 3.3 The Sturmian sequence $S_{\alpha, \rho}$ is a fixed point of some non-trivial invertible substitution if and only if $S_{\alpha, \rho}^{\prime}$ is also.

\section{Characterization of substitutive Sturmian sequences}

In this section, we give the proof of the following characterization which is due to Yasutomi [10].

Theorem 4.1 Let $\alpha \in[0,1]$ be irrational, $\rho \in[0,1], \eta$ be the Sturmian sequence with slope $\alpha$ and intercept $\rho$. Then $\eta$ is a fixed point of some non-trivial substitution if and only if

- $\alpha$ is a Sturm number, i.e. a quadratic irrational with $\bar{\alpha} \notin(0,1)$;

- $\rho \in \mathbb{Q}(\alpha)$, and $\bar{\rho}$ is between $\bar{\alpha}$ and $1-\bar{\alpha}$

Proof: We first prove the necessity.

Obviously, we have that $\alpha$ is a quadratic irrational, and $\lambda \in \mathbb{Q}(\alpha)$ by Lemma 3.1. Taking complexconjugate in Eq. 11 yields $M\left(\begin{array}{c}1-\bar{\alpha} \\ \bar{\alpha}\end{array}\right)=\bar{\lambda}\left(\begin{array}{c}1-\bar{\alpha} \\ \bar{\alpha}\end{array}\right)$, so $(\bar{\alpha}, \bar{\alpha}-1) M=\lambda(\bar{\alpha}, \bar{\alpha}-1)$. By PerronFrobenius Theorem, $\bar{\alpha}>1$ or $\bar{\alpha}<0$, that is $\alpha$ is a Sturm number.

By Theorem 3.2. $\rho=1-\alpha-\frac{1}{1-\bar{\lambda}}(|u| \alpha-\lfloor(|u|+1) \alpha\rfloor) \in \mathbb{Q}(\alpha)$ and

$$
\bar{\rho}=1-\bar{\alpha}+\frac{1}{\lambda-1}(|u| \bar{\alpha}-\lfloor(|u|+1) \alpha\rfloor) .
$$

Regarding as a function of $|u|, \bar{\rho}$ is strictly increasing if $\bar{\alpha}>1$; and is strictly decreasing if $\bar{\alpha}<0$. Since $0 \leq|u| \leq\|M\|-2$, and if $|u|=0, \rho=1-\alpha$ and $\bar{\rho}=1-\bar{\alpha}$; if $|u|=\|M\|-2, \rho=\alpha$ and $\bar{\rho}=\bar{\alpha}$. So $\bar{\rho}$ is between $\bar{\alpha}$ and $1-\bar{\alpha}$. This completes the proof of the necessity.

To prove the sufficiency, we need the following Theorem of Crisp, Moran, Pollington \& Shiue [3]:

Theorem 4.2 The characteristic word $C_{\alpha}$ is fixed by some non-trivial substitution iff $\alpha$ is a Sturm number.

Now we continue the proof. Suppose $(\alpha, \rho)$ satisfies the conditions; $\eta$ is the corresponding Sturmian sequence. Since $\alpha$ is Sturm, there exists $\tau$ which fixes $C_{\alpha}$ by the preceding theorem. And replacing $\tau^{2}$ by $\tau$ if necessary, we assume that $\operatorname{det} M=1$.

We will show that $\eta$ is a fixed point of a non-trivial substitution by writing $\rho$ in a suitable form.

Consider firstly the case that $\rho \in 1-\alpha-\frac{1}{1-\bar{\lambda}} \mathbb{Z}[\alpha]$, say $\rho=1-\alpha-\frac{1}{1-\bar{\lambda}}(-m+n \alpha)(m, n \in \mathbb{Z})$.

Since $\rho \in[0,1]$, we have

$$
-m+n \alpha \in(1-\bar{\lambda})[-\alpha, 1-\alpha] \subset(-\alpha, 1-\alpha)
$$


here ' $\subset$ ' is due to the facts that $0 \in[-\alpha, 1-\alpha]$ and $0<1-\bar{\lambda}<1$. This implies that $m=\lfloor(n+1) \alpha\rfloor$.

Hence, $\bar{\rho}=1-\bar{\alpha}+\frac{1}{\lambda-1}(n \bar{\alpha}-\lfloor(n+1) \alpha\rfloor)$. Again $\bar{\rho}$ is strictly monotone as a function of $n$. By arguing as in the proof of the necessity, $\bar{\rho}(0)=1-\bar{\alpha}$ and $\bar{\rho}(\|M\|-2)=\bar{\alpha}$. Then from the range of $\bar{\rho}$, we get

$$
n \in\{0,1, \cdots,\|M\|-2\} .
$$

This means that $\eta$ is a fixed point of $\tau_{n}$ in the conjugate class of $\tau$ (See Eq.(4)).

In general, if $\rho \in 1-\alpha-\frac{1}{1-\bar{\lambda}^{K}} \mathbb{Z}[\alpha]$ for some $K \in \mathbb{Z}^{+}$, the same argument (with $\tau^{K}$ in place of $\tau$ ) shows that $\eta$ is a fixed point of some substitution in the conjugate class of $\tau^{K}$. So it suffices to show $\rho \in 1-\alpha-\frac{1}{1-\bar{\lambda}^{K}} \mathbb{Z}[\alpha]$ for some $K \in \mathbb{Z}^{+}$. Now we will show that for any $\rho \in \mathbb{Q}(\alpha)$ there exist an integer $K$ such that $\rho \in 1-\alpha-\frac{1}{1-\bar{\lambda}^{K}} \mathbb{Z}[\alpha]$.

Put $\beta:=1-\alpha-\rho \in \mathbb{Q}(\alpha)$. Take $p \in \mathbb{Z}^{+}$such that $p \beta \in \mathbb{Z}[\alpha]$. From Eq. $[1]$, we have $\lambda \mathbb{Z}[\alpha] \subset \mathbb{Z}[\alpha]$. Since $\lambda+\bar{\lambda} \in \mathbb{Z}, \bar{\lambda} \mathbb{Z}[\alpha] \subset \mathbb{Z}[\alpha]$. Thus $\bar{\lambda}^{n} \in \mathbb{Z}[\alpha]$.

By Pigeonhole Principle, there exist $m<m^{\prime}$ such that $\bar{\lambda}^{m}-\bar{\lambda}^{m^{\prime}} \in p \mathbb{Z}[\alpha]$. Since $\lambda \bar{\lambda}=\operatorname{det} M=1$, $1-\bar{\lambda}^{m^{\prime}-m} \in p \lambda^{m} \mathbb{Z}[\alpha] \subset p \mathbb{Z}[\alpha]$.

Putting $K=m^{\prime}-m$, we have

$$
\left(1-\bar{\lambda}^{K}\right) \beta \in \mathbb{Z}[\alpha],
$$

and this concludes the proof of the sufficiency.

\section{References}

[1] P. Baláži, Z. Masáková, and E. Pelantová. Complete characterization of substitution invariant Sturmian sequences. Integers, 5(1):A14, 23 pp. (electronic), 2005.

[2] V. Berthé, H. Ei, S. Ito, and H. Rao. On substitution invariant Sturmian words: an application of Rauzy fractals. Theor. Inform. Appl., 41(3):329-349, 2007.

[3] D. Crisp, W. Moran, A. Pollington, and P. Shiue. Substitution invariant cutting sequences. J. Théor. Nombres Bordeaux, 5(1):123-137, 1993.

[4] H. Ei and S. Ito. Decomposition theorem on invertible substitutions. Osaka J. Math., 35(4):821-834, 1998.

[5] N. Pytheas Fogg. Substitutions in dynamics, arithmetics and combinatorics, volume 1794 of Lecture Notes in Mathematics. Springer-Verlag, Berlin, 2002. Edited by V. Berthé, S. Ferenczi, C. Mauduit and A. Siegel.

[6] M. Lothaire. Algebraic combinatorics on words, volume 90 of Encyclopedia of Mathematics and its Applications. Cambridge University Press, Cambridge, 2002.

[7] F. Mignosi and P. Séébold. Morphismes sturmiens et règles de Rauzy. J. Théor. Nombres Bordeaux, 5(2):221233, 1993.

[8] B. Tan and Z.-Y. Wen. Invertible substitutions and Sturmian sequences. European J. Combin., 24(8):983-1002, 2003.

[9] Z.-X. Wen and Z.-Y. Wen. Local isomorphisms of invertible substitutions. C. R. Acad. Sci. Paris Sér. I Math., 318(4):299-304, 1994.

[10] S. Yasutomi. On Sturmian sequences which are invariant under some substitutions. In Number theory and its applications (Kyoto, 1997), volume 2 of Dev. Math., pages 347-373. Kluwer Acad. Publ., Dordrecht, 1999. 